\title{
Chapter 14 \\ Focused Proteomics on Egg Membrane Microdomains to Elucidate the Cellular and Molecular Mechanisms of Fertilization in the African Clawed Frog Xenopus laevis
}

\author{
Ken-ichi Sato, A.K.M. Mahbub Hasan, and Takashi W. Ijiri
}

\begin{abstract}
Involvement of protein tyrosine kinase-dependent signal transduction (PTK signaling) in fertilization was initially demonstrated by studies using sea invertebrates: namely, an increase of tyrosine phosphorylation in egg or embryo proteins is shown to occur within minutes after gamete interaction. Among vertebrate species so far studied are fish, frog, and some mammalian species in which the importance of PTK signaling for fertilization or activation of development has been shown. In this review chapter, we summarize our experimental data that explore the role played by the tyrosine kinase Src in fertilization of the African clawed frog Xenopus laevis. In addition, we introduce our recent approaches that focus on the structure and function of egg membrane microdomains (MDs), where the Src PTK signaling machinery is organized. Finally, we propose a hypothesis that gamete membrane interaction at fertilization is accompanied by mutual signaling cross-talk between egg and sperm using the egg MDs as scaffolds and discuss the versatility of our hypothesis in general understanding of the sexual reproduction mechanism.
\end{abstract}

Keywords Cross-talk between gametes - In vitro reconstitution - Membrane microdomains $\bullet$ Signal transduction $\bullet$ Tyrosine phosphorylation

K. Sato $(\bowtie) \bullet$ T.W. Ijiri

Laboratory of Cell Signaling and Development, Department of Molecular Biosciences,

Faculty of Life Sciences, Kyoto Sangyo University, Kyoto 603-8555, Japan

e-mail: kksato@cc.kyoto-su.ac.jp

A.K.M. Mahbub Hasan

Laboratory of Cell Signaling and Development, Department of Molecular Biosciences,

Faculty of Life Sciences, Kyoto Sangyo University, Kyoto 603-8555, Japan

Laboratory of Gene Biology, Department of Biochemistry and Molecular Biology,

University of Dhaka, Dhaka 1000, Bangladesh 


\subsection{Src PTK Signaling and Fertilization}

Protein tyrosine phosphorylation was initially discovered as an intracellular phenomenon to be associated with malignancy of cancer cells (Hunter 2009). The discovery was made in the process of research on the molecular function of a gene product of Rous sarcoma virus, that is, v-Src, the memorably first example of socalled cancer gene or oncogene. Such a breakthrough finding, in conjunction with another epoch-making discovery of the first example of cellular cancer gene or proto-oncogene, c-Src (hereafter Src), has led many researchers to study the physiological and pathological importance of protein tyrosine phosphorylation catalyzed by Src and other protein tyrosine kinases (PTKs) (e.g., Abelson kinase, epidermal growth factor receptor/kinase, insulin receptor/kinase) (Abram and Courtneidge 2000; Hunter and Cooper 1985; Jove and Hanafusa 1987; Thomas and Brugge 1997). Now, it is well established that a variety of cellular functions involve this kind of posttranslational modification of proteins. Under this background, fertilization is one of the earliest and pioneering as well as contemporary subjects in the PTK research field (Kinsey 2013; Sato et al. 2004; Sato 2008).

Dasgupta and Garbers (1983) published the first report on protein tyrosine phosphorylation in the fertilization study: they demonstrated that PTK activity toward synthetic peptide substrates is present in unfertilized sea urchin eggs and continues to increase during early embryogenesis. Given that early embryos of sea urchin, similar to rapidly proliferating cancer cells, undergo several cycles of very fast cell division, that is, cleavage, it seems to be natural that they employ high PTK activity. Further detailed studies by Kinsey's group and some other researchers (including us) using sea urchins and other animal species, however, highlighted facts of specific importance in this cellular system, that PTK activity is rapidly and transiently activated in response to fertilization, or more precisely, gamete interaction (Abassi and Foltz 1994; Ciapa and Epel 1991; Kamel et al. 1986; Ribot et al. 1984; Sato et al. 1996; Wu and Kinsey 2000), and that the activated PTK may be responsible for sperm-induced increase(s) in calcium concentrations within the fertilized eggs, whose occurrence is believed to be indispensable for the subsequent initiation of embryonic development, in other words, "egg activation" (Giusti et al. 1999; Kinsey and Shen 2000; Runft and Jaffe 2000; Sato et al. 2000).

As described, compelling evidence suggests that egg-associated PTK signaling serves an important role in sperm-induced egg activation of nonmammalian species. As well appreciated in somatic cell systems, PTK signaling usually requires the binding of extracellular ligands to their cell-surface receptors. Therefore, it has been thought that gamete interaction at fertilization may act as a ligand-like signal to stimulate an egg-surface receptor so that the intracellular PTK signaling is triggered. On the other hand, eggs of mammalian species (e.g., mouse) and some other nonmammalian species (e.g., bird, newt) seem to employ sperm-derived factor(s), which would be incorporated into the egg cytoplasm, for the sperm-induced calcium responses: molecules identified so far include phospholipase C $\zeta$ (Swann and Lai 2013) and citrate synthase (Iwao 2012). This observation may reflect that gamete membrane interaction-mediated and gamete membrane fusion-mediated egg activation systems employ their specific molecular machinery and, perhaps more importantly, some other 
differences of fundamental importance that are found between these species: aquatic or terrestrial life, and external or internal fertilization.

In this review article, we explain why we analyze egg membrane microdomains (MDs) and summarize briefly our achievements, with a special focus on the finding of the egg MDs as structural and functional platform for sperm-induced Src PTK signaling. Then, we introduce our current research projects, evaluating the hypothesis that Src and the other MD-associated molecules constitute a signaling network for successful gamete interaction and activation of development. About 10 years ago, we published one review article in the journal Proteomics on our focused proteomics project on Xenopus egg MDs (Sato et al. 2002a). Therefore, a part of this review article can be considered as updated information for the MDs projects.

\subsection{Characterization of Src as a Mediator of Gamete Interaction and Egg Activation}

It was 1996 when we published the first paper on the Xenopus egg Src (hereafter $\mathrm{xSrc}$ ) and its possible involvement in fertilization signaling (Sato et al. 1996). In that paper, we showed data about chromatographic fractionation of membrane-associated proteins and in vitro protein kinase assay, by which we could detect an elevation of the activity of xSrc in response to fertilization. Our further studies demonstrated that pharmacological (e.g., use of inhibitors; Sato et al. 1999, 2000, 2001) or molecular biological inhibition of xSrc (i.e., expression of kinase-negative mutant of xSrc; manuscript in preparation) impairs the ability of Xenopus eggs to undergo calcium reactions and egg activation in response to sperm (Sato et al. 1999) and that Xenopus eggs can be activated in a Src-dependent manner by artificial egg activators that interacts with the egg surface (i.e., RGD peptide and cathepsin B; Sato et al. 1999; Mahbub Hasan et al. 2005) and by hydrogen peroxide that may directly activate xSrc (Sato et al. 2001). These results suggest that xSrc acts between gamete interaction/fusion and an increase in intracellular calcium concentration at fertilization (Fig. 14.1). In support of this, phospholipase $C \gamma$, whose activation leads to the hydrolysis of phosphatidylinositol-4,5-bisphosphates and production of the intracellular second messengers diacylglycerol and inositol-1,4,5-trisphosphate (direct activator for intracellular calcium release from endoplasmic reticulum), was shown to be a substrate of the activated xSrc (Sato et al. 2000, 2001, 2003).

Other targets of the activated xSrc include Shc (Aoto et al. 1999), hnRNP K (Iwasaki et al. 2008), lipovitellin 2 (Kushima et al. 2011), and pp40, whose identity is not yet demonstrated (manuscript in preparation) (Fig. 14.1). All these proteins and PLC $\gamma$ are, however, cytoplasmic proteins resembling $\mathrm{xSrc}$, so that they could not be directly involved in the gamete interaction and subsequent Src activation. Given that $\mathrm{xSrc}$ seems to be involved in the upstream signaling for egg activation and perhaps other cellular functions for embryonic development (e.g., translational control of maternal mRNAs), our goal has shifted to understand the mechanisms of gamete interaction and subsequent $\mathrm{xSrc}$ activation. Under these circumstances, we became interested in analyzing the egg plasma membrane, or more specifically, 


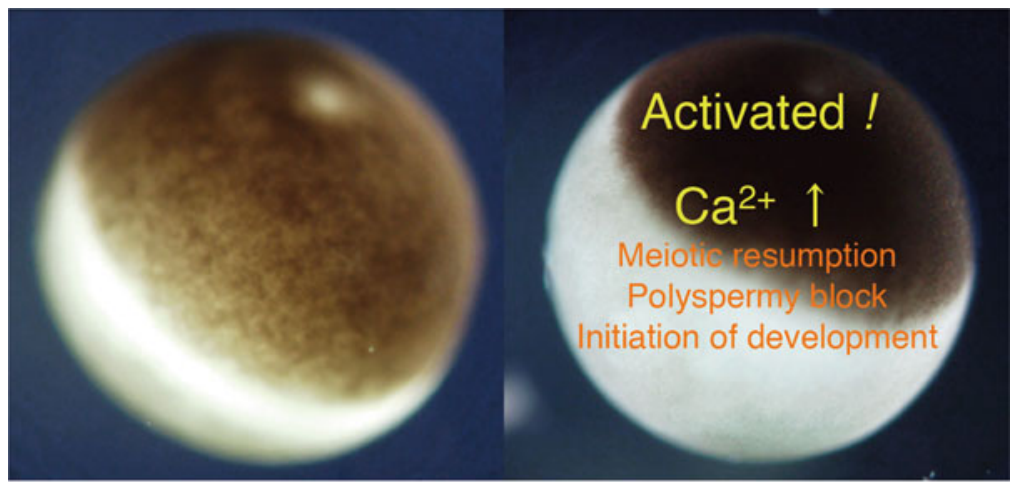

\section{Unfertilized egg $\longrightarrow \quad$ Fertilized egg}

\section{Tyrosine kinase-dependent signal transduction}

Fig. 14.1 Molecular mechanisms of fertilization and activation of development in Xenopus laevis. Shown are photographs for a X. laevis egg before (left) and after (right) fertilization. Note that cortical contraction in the pigmented area of the egg (animal hemisphere) is evident after fertilization. Successful gamete membrane interaction and fusion leads to a transient increase in intracellular $\mathrm{Ca}^{2+}$ concentration in the egg, by which a series of events, collectively called "egg activation," takes place. The egg activation events include meiotic resumption, block to polyspermy, nuclear fusion, and initiation of embryonic development. Studies from our research group and others have demonstrated that tyrosine kinase-dependent signal transduction serves a pivotal role in connecting gamete membrane interaction and $\mathrm{Ca}^{2+}$-dependent egg activation

membrane microdomains and their associated molecules. In the following sections, we introduce membrane microdomains and describe our achievements were obtained by the study on this subject.

\subsection{Focused Proteomics on Xenopus Egg MDs: Achievements and Problems}

\subsubsection{Rationale to Study MDs for Exploring the Mechanism of Fertilization}

A growing body of knowledge indicates that cellular plasma membranes consist of mixtures of heterogeneously organized substructures, whose specific identities depend on the composition of lipids, proteins, and their associated carbohydrates (Brown and London 1998; Simons and Ikonen 1997; Simons and Sampaio 2011). These membrane substructures are also called as membrane subdomains or microdomains: we prefer to use the term "microdomains" (MDs) because it would reflect the real scale of these membrane substructures (i.e., diameters of submicrometers or a few micrometers) (Pike 2006). 
Experimentally, conventional biochemical fractionation methods are used to isolate MDs; hallmarks for obtaining MDs are such criteria as insolubility under certain cell extraction methods (e.g., resistance to detergent extraction) and tendency to float under ultracentrifugation (i.e., low density). The resulting low-density and detergent-insoluble membranes (LD-DIMs) are often regarded as lipid/membrane "rafts" and are enriched in cholesterol and some specific subsets of components (e.g., sphingolipids, gangliosides, signaling proteins). Several lines of evidence indicate that MDs constitute a platform/scaffold for capturing/sensing extracellular signals (e.g., growth factors, environmental stimuli), as well as for transmitting the signals into the cytoplasm and nucleus, by which a variety of cellular functions are exerted (Simons and Ikonen 1997; Simons and Toomre 2000).

In the fertilization research field, Kitajima and colleagues demonstrated first the presence of the sea urchin sperm MDs and their possible involvement in fertilization (Ohta et al. 1999). Publication of this leading report and our data showing that Xenopus egg fertilization involves Src PTK signaling (see foregoing) led us to examine the presence and physiological importance of MDs in Xenopus eggs. For a more detailed introduction to MDs, please also refer to our recent publication (Mahbub Hasan et al. 2011).

\subsubsection{Xenopus Egg MDs Projects: Achievements and Problems}

\subsubsection{Discovery of Egg MDs as an Important Resource for Fertilization Study}

In 2002, we published the first report on Xenopus egg MDs (Sato et al. 2002b), in which we demonstrated the following: (1) extraction of unfertilized eggs in the presence of Triton X-100 and subsequent ultracentrifugation under stepwise gradients of sucrose concentration yield LD-DIMs fractions (see above) that are enriched in cholesterol, the GM1 ganglioside, and most importantly for us, xSrc; (2) the LD-DIMs fractions that are prepared from fertilized eggs contain at least three tyrosine-phosphorylated proteins, two of which are XSrc and PLC $\gamma$; (3) a similar and more augmented pattern of protein tyrosine phosphorylation is seen in the LD-DIMs that are prepared from hydrogen peroxide-activated eggs; and (4) in the LD-DIMs of eggs that are activated by the calcium ionophore A23187, no increase in protein tyrosine phosphorylation is observed. These results suggest that the LD-DIMs fractions contain MDs, in which sperm-induced Src PTK signaling is operating (Fig. 14.1).

The functional importance of MDs in fertilization is suggested by the studies using methyl- $\beta$-cyclodextrin $(\mathrm{M} \beta \mathrm{CD})$, a drug that causes disruption of the cholesteroldependent membrane structures. We found that unfertilized Xenopus eggs that are preincubated with this substance fail to undergo tyrosine phosphorylation of the LD-DIMs-associated proteins, intracellular calcium release, and other egg activation events (Sato et al. 2002b). Such inhibitory effect of M $\beta C D$ could be canceled by the addition of excess amounts of cholesterol; therefore, it was not simply caused by 
a

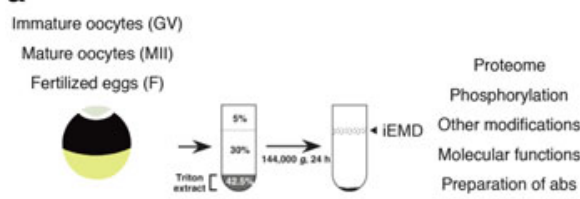

C

a.
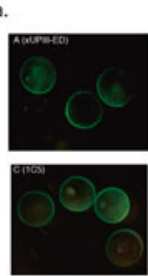

b

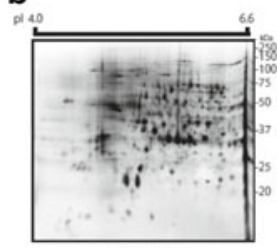

GV-MD

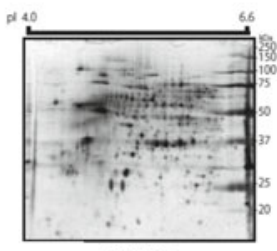

MII-MD

d

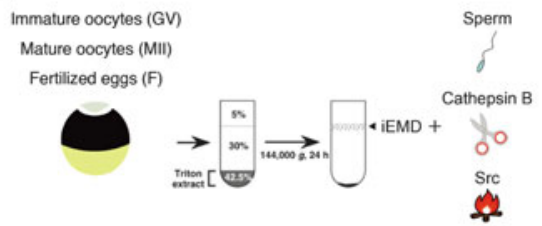

Fig. 14.2 Xenopus egg microdomains (MDs) project. (a) Our ongoing snapshot-type MDs project includes conventional, differential proteome analysis (b shows example images), analysis of posttranslational modifications such as phosphorylation, molecular targeting analysis, and preparation and characterization of monoclonal antibody library (c shows example images). These experiments involve the use of MDs that are isolated from fully grown, immature oocytes at the germinal vesicle stage (GV oocytes), mature and unfertilized oocytes/eggs at the second meiotic metaphase (MII oocytes), and fertilized embryos, all of which are called iEMD (isolated egg MDs). (b) Proteome patterns for MDs-associated proteins in GV oocytes (left) and MII oocytes (right), both of which are separated by two-dimensional gel electrophoresis (first dimension, isoelectric focusing at the $\mathrm{pI}$ range of 4.0-6.6; second dimension, SDS-polyacrylamide gel electrophoresis) and visualized by silver staining. (c) In panels shown in $a$, indirect immunofluorescent experiments are performed to verify the binding of monoclonal antibodies to the surface of unfertilized Xenopus eggs. In $b$, the binding of the antibody to egg proteins is examined by immunoblotting. (d) Our ongoing MDs project also includes in vitro reconstitution of signal transduction events associated with fertilization. In this project, iEMD are subjected to in vitro treatment with sperm, cathepsin B, Src, or some others, and analyzed for their biochemical (e.g., tyrosine phosphorylation of MDsassociated proteins) as well as cell biological responses (e.g., ability of the sperm to fertilize eggs)

toxicity of the substance. Moreover, we found that the addition of sperm to the LD-DIMs, which were isolated from unfertilized eggs, caused an increase in tyrosine phosphorylation of proteins that are present in the LD-DIMs. These results argue the idea that LD-DIMs contain MDs, in which both the receptor for sperm and the signaling machinery for sperm-induced Src activation are pre-organized.

The aforementioned data have led us to consider two major directions of the MDs project (Sato et al. 2002a) (Fig. 14.2). The first subproject is to identify novel fertilization-related components by characterization of MDs-associated molecules. A major achievement in this subproject is the identification of a type I transmembrane protein uroplakin III (UPIII) that is thought to be involved in gamete interaction, 
which may involve the action of sperm-derived protease, and regulation of $\mathrm{xSrc}$ activity, in that UPIII may be involved in the negative regulation of xSrc in unfertilized eggs (Mahbub Hasan et al. 2005, 2007; Mammadova et al. 2009; Sakakibara et al. 2005a) (Fig. 14.1). The second subproject is to examine in vitro reconstitution of the fertilization signaling events with the use of isolated MDs. We have succeeded in reconstituting sperm-induced egg activation events such as tyrosine phosphorylation of xSrc and PLC $\gamma$, calcium responses, and resumption of the meiotic cell cycle by using isolated, unfertilized egg MDs and cytostatic factor-arrested unfertilized egg extracts (Sato et al. 2003, 2006). In the remaining part of this subsection, we describe achievements and current problems in these two subprojects.

\subsubsection{Characterization of UPIII as a Novel Component of Fertilization}

UPIII was originally identified by mass spectrometric analysis of a prominently tyrosine-phosphorylated 30-kDa protein that is present in the LD-DIMs fractions of fertilized eggs (for more detail on UPIII and other UP family proteins, see Mahbub Hasan et al. 2011). Coexpression of UPIII and XSrc in human embryonic kidney cells results in tyrosine phosphorylation of UPIII. These results suggest that the cytoplasmic part of UPIII acts as a target of xSrc in fertilized Xenopus eggs, although physiological relevance of the phosphorylation is unknown. It was also shown that treatment of Xenopus eggs with cathepsin B, a mimetic enzyme of the spermderived protease that can promote parthenogenetic activation of the eggs, causes partial degradation of UPIII and activation of xSrc. These two events were inhibited when eggs are pretreated with an antibody that is raised against the extracellular domain of UPIII. More importantly, the same antibody also effectively inhibits sperm-induced egg activation. These results suggest that the extracellular part of UPIII acts as a sperm receptor, by which it transmits the sperm signal into the egg cytoplasm (via xSrc activation) (Fig. 14.3). One potential mechanism of UPIII-xSrc connection is that, in unfertilized Xenopus eggs, the molecular complex consisting of UPIII and UPIb, a well-known binding partner of UPIII, suppresses the activity of $\mathrm{xSrc}$, and that the proteolysis of UPIII at fertilization leads to the liberation of the activated xSrc. This idea is suggested by studies using the overexpression systems of culture cells (Mahbub Hasan et al. 2007).

\subsubsection{In Vitro Reconstitution of Fertilization Signaling by Isolated MDs}

Reportedly, cellular and molecular insights into the cell-cycle events (e.g., mitosis, meiotic resumption) have been well documented by studies using cell-free extracts that are prepared from unfertilized Xenopus eggs (Murray 1991). In particular, cytostatic factor-arrested unfertilized egg extracts (CSF extracts) are used to reconstitute meiosis and other cell-cycle events, as seen in Xenopus oocytes or embryos (Maresca and Heald 2006; Ohsumi et al. 2006). In this experimental 


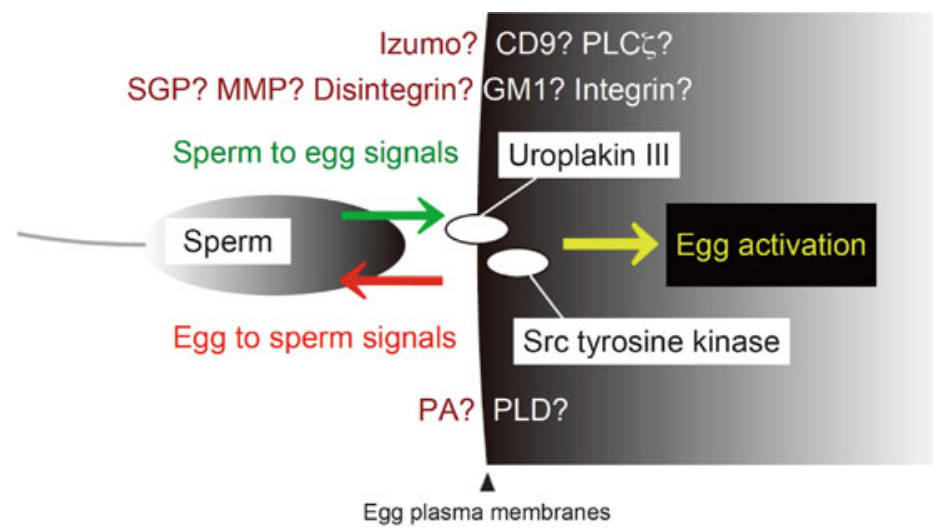

Fig. 14.3 Signaling cross-talk between sperm and egg MDs. A number of gamete membraneassociated components have been implicated in fertilization, some of which seem to be species specific whereas others are of universal importance in a wide variety of species. In Xenopus laevis, sperm-induced egg activation involves proteolytic cleavage of UPIII and activation of the tyrosine kinase $\mathrm{xSrc}$, both of which are localized events in the egg MDs. Our recent data suggest that membrane interaction between egg and sperm via the egg MDs also involves modulation of fertilizing property in sperm. Such bidirectional signaling between egg and sperm may be crucial for completion of successful gamete interaction/fusion and initiation of embryonic development

platform, however, the only events to be evaluated are cytoplasmic and nuclear functions. Our idea in the second MDs subproject is to employ the isolated MDs as a resource to reconstitute plasma membrane-associated functions and their interactions with cytoplasmic environments at fertilization (Fig. 14.2). As described earlier, the addition of sperm to the isolated MDs causes an elevation in tyrosine phosphorylation of MDs-associated proteins. This phenomenon actually involves the activation of xSrc as well as phosphorylation of UPIII (Sato et al. 2003, 2006; unpublished results), both of which are the earliest events that are seen in fertilized Xenopus eggs. Therefore, we sought to combine the sperm-treated MDs with CSF extracts to reconstitute events of fertilization from the plasma membranes through the cytoplasm. As a result, we have found that the sperm-treated MDs can promote a transient calcium release, dephosphorylation of mitogen-activated protein kinase, and cell-cycle progression as judged by the morphological change in sperm-derived nuclei (Sato et al. 2003). In addition, the isolated MDs that are pretreated with hydrogen peroxide (Src PTK signaling is stimulated), but not those pretreated with A23187 (Src PTK signaling is not stimulated), are shown to promote the aforementioned events. These results are consistent with the idea that egg MDs can be used as an experimental platform to reconstitute membrane-associated, Src PTKdependent signaling events at fertilization (Sato et al. 2003).

Given this background, there are two major problems to be solved in our MDs project: one is when and how sperm-dependent signaling functions of MDs are established; and the other is how egg MDs interact with sperm and what is the consequence of this interaction. 


\subsubsection{Ongoing Approaches to Explore the Physiological Functions of MDs}

As already described, our MDs project has involved two major subprojects: snapshot analysis of MDs-associated proteins and in vitro reconstitution of MDs functions (Fig. 14.2), and these two subprojects have raised new problems and questions. To explore further these problems and questions, we are now undergoing a panel of experiments. In the following subsections, we describe the aims and current states of those ongoing MDs projects.

\subsubsection{Evaluation of UPIII and MDs Functions in Immature Oocytes}

In general, ovarian and immature oocytes cannot be fertilized because they are not yet fully competent to undergo sperm-induced developmental activation. It has long been appreciated that hormone-induced oocyte maturation is an event in that oocytes acquire fertilization competence by establishing intracellular conditions (e.g., meiotic cell-cycle stage, subcellular localizations of endoplasmic reticulum and other intracellular components, biochemical properties including the activity of certain protein kinases such as maturation-promoting factor). However, it seems that oocyte maturation also involves an alteration in the structure and function of the oocyte plasma membranes. To access this possibility, we are now analyzing expression, subcellular localization, and molecular interaction of UPIII in the course of oocyte growth (oogenesis) and progesterone-induced oocyte maturation. In addition, we are examining whether sperm-induced PTK signaling can work in the immature oocyte MD. Results so far obtained suggest that oocyte maturation leads to the exposure of the extracellular domain of UPIII on the oocyte surface and that, at a similar timing, MDs become fully competent for sperm-induced PTK signaling (manuscript in preparation).

\subsubsection{Gain- and Loss-of-Function Experiments on xSrc and UPIII}

We have prepared immature oocytes expressing xSrc of either kinase-active or kinase-negative mutants, and we are now examining in vitro maturation of the oocytes and their activation in response to artificial egg activators: the calcium ionophore A23187, cathepsin B, and hydrogen peroxide (manuscript in preparation). We have also constructed mutant UPIII, in which its possible target amino acids for tryptic protease in the extracellular domain are mutated to be proteolysis resistant. We are now characterizing molecular function of the mutant UPIII (named UPIIIRRAA mutant) by using expression systems with human embryonic kidney 293 cells. Results so far obtained demonstrate that the UPIII-RRAA mutant, as does the wild-type UPIII, localizes to plasma membranes/MDs and is capable of inactivating the coexpressed xSrc in 293 cells (manuscript in preparation). In addition, more importantly, it has been shown that the UPIII-RRAA is actually resistant to 
proteolysis by cathepsin B. We are now examining the sperm-induced PTK signaling function of MDs that are prepared from 293 cells expressing the UPIII of either the wild type or RRAA mutant. These experiments would not only evaluate the function of the UPIII-RRAA mutant but also suggest that the sperm receptor function can be reconstituted in MDs of the cultured cells.

\subsubsection{Unbiased Approaches to Identify and Characterize Novel Components}

Although the aforementioned two approaches focus on functions of certain well-characterized egg MD-associated proteins (i.e., xSrc and UPIII), the following two approaches are aimed to discover novel fertilization-related components that localize to the egg MDs. The first one is to generate a library of monoclonal antibodies in which plasma membrane-associated components are used as antigens. In Xenopus, some egg surface-interacting substances have been reported to act as an egg activator: examples include disintegrin peptides (Iwao and Fujimura 1996; Shilling et al. 1998) and cathepsin B (Mizote et al. 1999). As we mentioned earlier, an antibody that recognizes the extracellular domain of UPIII has been shown to inhibit normal fertilization (Sakakibara et al. 2005a). Therefore, we expect that an antibody(s) of the library, which can bind to the surface (in other words, some known or unknown components essential for fertilization) of unfertilized Xenopus eggs, would be able to activate or inhibit egg functions. We are now screening monoclonal antibody clones that satisfy the following criteria: (1) to bind to the egg surface in indirect immunofluorescent experiments, (2) to bind to protein bands in immunoblotting experiments, and (3) to inhibit normal fertilization or to activate the egg in the absence of sperm. The second approach is to identify the molecular basis of polarity in the egg surface. In Xenopus, sperm entry point is limited to somewhere in the pigmented area of the egg: the animal hemisphere. Our hypothesis is that this limitation is the result of uneven distribution of sperm receptor or its inhibitory machinery. To access this problem, we are now undergoing comparative analysis of the animal and vegetal hemisphere-associated proteins. Other kinds of differential proteome analysis have been done in our previous study, in that an antibody that recognizes fertilization-induced plasma membrane-associated antigens in Xenopus eggs is generated by subtractive immunization (Sakakibara et al. 2005b). Therefore, these two ongoing experiments would also be useful for further evaluation of such an "unbiased" approach to discover fertilization-related egg components.

\subsubsection{Analysis of Signaling Cross-Talk Between MDs and Sperm or Egg Cytoplasm}

In this ongoing project, we analyze the interactions of the egg MDs with sperm and the egg cytoplasm. Regarding the egg MD-sperm interactions, we have preliminary data showing that the isolated egg MDs do not inhibit normal 
fertilization when they are present in the insemination media, but rather recover the ability of sperm to fertilize the eggs that are pretreated with the anti-UPIII extracellular domain antibody (manuscript in preparation). In other words, it is suggested that the MD-treated sperm somehow overcome the absence of functional UPIII on the egg surface. In Xenopus, no evidence is available with regard to the presence of extracellular MDs- or other membrane-containing vesicles (such as exosomes in the mouse; Miyado et al. 2008; manuscript in preparation). Therefore, we assume that, exactly at the time of gamete membrane interaction and fusion, egg-"associated" MDs act not only as a platform for sperm-induced Src PTK signaling in fertilized eggs but also as an unknown signaling trigger for sperm, by which sperm may acquire the ability to fertilize eggs, in a UPIIIdependent manner (Fig. 14.3). We now assess this "bidirectional signaling between sperm and the egg MDs" hypothesis by analyzing what kind of cellular functions in sperm are modulated after their interactions with the isolated MDs, and what kind of molecule(s) in the sperm surface are involved in the interactions with the isolated MDs. One interesting thought is the involvement of PTK signaling in sperm, which has been well documented in several other aspects of sperm functions (Ijiri et al. 2012).

\subsubsection{Analysis of Signaling Cross-Talk Between MDs and Egg Mitochondria}

We are also interested in functional interactions between egg MDs and organelles such as mitochondria. This interest is based on the fact that serine/threoninespecific protein kinase Akt, whose activation is shown in fertilized Xenopus eggs (Mammadova et al. 2009), localizes predominantly to mitochondria and that two mitochondrion-associated proteins of $\sim 40 \mathrm{kDa}$ on SDS-polyacrylamide gel electrophoresis (named pp40) are identified as possible Src substrates at fertilization (manuscript in preparation). These two proteins are also present in MD fractions. In some cell systems, cell-surface receptor/kinases [e.g., epidermal growth factor receptor (EGFR)] and membrane-associated cytoplasmic protein kinases (e.g., Akt, Src) are shown to translocate or localize to mitochondria in response to cell stimuli (Demory et al. 2009; Hebert-Chatelain 2013). Therefore, it will be interesting to examine whether fertilization involves a similar signaling linkage between MDs and mitochondria by way of characterization of these novel Src substrates. Our interest in mitochondria also involves a possible relationship of their known functions and fertilization. In somatic cell systems, mitochondria serve a pivotal role in ATP production and survival control. Thus, this ongoing project will explore the presence of MDs-mitochondria interactions at fertilization and their physiological relevance to the metabolism and viability of developing embryos. In this connection, we have started to analyze quantitative and spatiotemporal regulation of ATP in oocytes and eggs during oocyte maturation and fertilization (Ijiri et al. 2014). 


\subsection{Summary and Perspectives}

Our ongoing egg-MDs project, consisting of snapshot experiments and in vitro reconstitution experiments, molecular targeting approaches and unbiased proteomics approaches, and cell biological approaches involving sperm and egg mitochondria; aim to understand how structure and function of egg MDs are developed and how egg MDs and MD-associated molecules contribute to successful fertilization and subsequent embryogenesis. We believe that the knowledge obtained with this project in itself would contribute to general understanding of the fertilization system. In addition, fertilization system involves, with no exception, membrane interaction and fusion between female and male gametes, in spite of a countless variety of species-specific differences in the fertilization system such as molecules of fertilization (e.g. requirement of Src PTK signaling), systems for egg activation (e.g., physiological monospermy and polyspermy), extracellular environment for fertilization (e.g., internal and external fertilization), and even animal and nonanimal fertilization. Therefore, our approach would be useful, at least in part, for fertilization studies on a wide range of other organisms.

Acknowledgments This work is supported by Grants-in-Aid on Innovative Areas (22112522, 24112714) from the Ministry of Education, Culture, Sports, Science, and Technology, Japan to K.S.

Open Access: This article is distributed under the terms of the Creative Commons Attribution Noncommercial License which permits any noncommercial use, distribution, and reproduction in any medium, provided the original author(s) and source are credited.

\section{References}

Abassi YA, Foltz KR (1994) Tyrosine phosphorylation of the egg receptor for sperm at fertilization. Dev Biol 164(2):430-443

Abram CL, Courtneidge SA (2000) Src family tyrosine kinases and growth factor signaling. Exp Cell Res 254(1):1-13

Aoto M, Sato K, Takeba S, Horiuchi Y, Iwasaki T, Tokmakov AA, Fukami Y (1999) A 58-kDa Shc protein is present in Xenopus eggs and is phosphorylated on tyrosine residues upon egg activation. Biochem Biophys Res Commun 258(2):265-270

Brown DA, London E (1998) Functions of lipid rafts in biological membranes. Annu Rev Cell Dev Biol 14:111-136

Ciapa B, Epel D (1991) A rapid change in phosphorylation on tyrosine accompanies fertilization of sea urchin eggs. FEBS Lett 295(1-3):167-170

Dasgupta JD, Garbers DL (1983) Tyrosine protein kinase activity during embryogenesis. J Biol Chem 258(10):6174-6178

Demory ML, Boerner JL, Davidson R, Faust W, Miyake T, Lee I, Hüttemann M, Douglas R, Haddad G, Parsons SJ (2009) Epidermal growth factor receptor translocation to the mitochondria: regulation and effect. J Biol Chem 284(52):36592-36604

Giusti AF, Carroll DJ, Abassi YA, Terasaki M, Foltz KR, Jaffe LA (1999) Requirement of a Src family kinase for initiating calcium release at fertilization in starfish eggs. J Biol Chem 274(41):29318-29322

Hebert-Chatelain E (2013) Src kinases are important regulators of mitochondrial functions. Int J Biochem Cell Biol 45(1):90-98 
Hunter T (2009) Tyrosine phosphorylation: thirty years and counting. Curr Opin Cell Biol 21(2):140-146

Hunter T, Cooper JA (1985) Protein-tyrosine kinases. Annu Rev Biochem 54:897-930

Ijiri TW, Mahbub Hasan AK, Sato K (2012) Protein-tyrosine kinase signaling in the biological functions associated with sperm. J Signal Transduct 2012:181560

Ijiri TW, Kishikawa J, Imamura H, Iwao Y, Yokoyama K, Sato K (2014) ATP imaging system in the Xenopus laevis oocyte. In: Sawada H, Inoue N, Iwano M (eds) Sexual reproduction in animals and plants. Springer, Heidelberg, pp 181-186

Iwao Y (2012) Egg activation in physiological polyspermy. Reproduction 144(1):11-22

Iwao Y, Fujimura T (1996) Activation of Xenopus eggs by RGD-containing peptides accompanied by intracellular $\mathrm{Ca}^{2+}$ release. Dev Biol 177(2):558-567

Iwasaki T, Koretomo Y, Fukuda T, Paronetto MP, Sette C, Fukami Y, Sato K (2008) Expression, phosphorylation, and mRNA-binding of heterogeneous nuclear ribonucleoprotein $\mathrm{K}$ in Xenopus oocytes, eggs, and early embryos. Dev Growth Differ 50(1):23-40

Jove R, Hanafusa H (1987) Cell transformation by the viral src oncogene. Annu Rev Cell Biol 3:31-56

Kamel C, Veno PA, Kinsey WH (1986) Quantitation of a src-like tyrosine protein kinase during fertilization of the sea urchin egg. Biochem Biophys Res Commun 138(1):349-355

Kinsey WH (2013) Intersecting roles of protein tyrosine kinase and calcium signaling during fertilization. Cell Calcium 53(1):32-40

Kinsey WH, Shen SS (2000) Role of the Fyn kinase in calcium release during fertilization of the sea urchin egg. Dev Biol 225(1):253-264

Kushima S, Mammadova G, Mahbub Hasan AK, Fukami Y, Sato K (2011) Characterization of lipovitellin 2 as a tyrosine-phosphorylated protein in oocytes, eggs and early embryos of Xenopus laevis. Zool Sci 28(8):550-559

Mahbub Hasan AK, Sato K, Sakakibara K, Ou Z, Iwasaki T, Ueda Y, Fukami Y (2005) Uroplakin III, a novel Src substrate in Xenopus egg rafts, is a target for sperm protease essential for fertilization. Dev Biol 286(2):483-492

Mahbub Hasan AK, Ou Z, Sakakibara K, Hirahara S, Iwasaki T, Sato K, Fukami Y (2007) Characterization of Xenopus egg membrane microdomains containing uroplakin Ib/III complex: roles of their molecular interactions for subcellular localization and signal transduction. Genes Cells 12(2):251-267

Mahbub Hasan AK, Fukami Y, Sato K (2011) Gamete membrane microdomains and their associated molecules in fertilization signaling. Mol Reprod Dev 78(10-11):814-830

Mammadova G, Iwasaki T, Tokmakov AA, Fukami Y, Sato K (2009) Evidence that phosphatidylinositol 3-kinase is involved in sperm-induced tyrosine kinase signaling in Хenopus egg fertilization. BMC Dev Biol 9:68

Maresca TJ, Heald R (2006) Methods for studying spindle assembly and chromosome condensation in Xenopus egg extracts. Methods Mol Biol 322:459-474

Miyado K, Yoshida K, Yamagata K, Sakakibara K, Okabe M, Wang X, Miyamoto K, Akutsu H, Kondo T, Takahashi Y, Ban T, Ito C, Toshimori K, Nakamura A, Ito M, Miyado M, Mekada E, Umezawa A (2008) The fusing ability of sperm is bestowed by CD9-containing vesicles released from eggs in mice. Proc Natl Acad Sci USA 105(35):12921-12926

Mizote A, Okamoto S, Iwao Y (1999) Activation of Xenopus eggs by proteases: possible involvement of a sperm protease in fertilization. Dev Biol 208(1):79-92

Murray AW (1991) Cell cycle extracts. Methods Cell Biol 36:581-605

Ohsumi K, Yamamoto TM, Iwabuchi M (2006) Oocyte extracts for the study of meiotic M-M transition. Methods Mol Biol 322:445-458

Ohta K, Sato C, Matsuda T, Toriyama M, Lennarz WJ, Kitajima K (1999) Isolation and characterization of low density detergent-insoluble membrane (LD-DIM) fraction from sea urchin sperm. Biochem Biophys Res Commun 258(3):616-623

Pike LJ (2006) Rafts defined: a report on the Keystone Symposium on Lipid Rafts and Cell Function. J Lipid Res 47(7):1597-1598

Ribot HD Jr, Eisenman EA, Kinsey WH (1984) Fertilization results in increased tyrosine phosphorylation of egg proteins. J Biol Chem 259(8):5333-5338 
Runft LL, Jaffe LA (2000) Sperm extract injection into ascidian eggs signals $\mathrm{Ca}^{2+}$ release by the same pathway as fertilization. Development (Camb) 127(15):3227-3236

Sakakibara K, Sato K, Iwasaki T, Kitamura K, Fukami Y (2005a) Generation of an antibody specific to Xenopus fertilized eggs by subtractive immunization. Genes Cells 10(4):345-356

Sakakibara K, Sato K, Yoshino K, Oshiro N, Hirahara S, Mahbub Hasan AK, Iwasaki T, Ueda Y, Iwao Y, Yonezawa K, Fukami Y (2005b) Molecular identification and characterization of Xenopus egg uroplakin III, an egg raft-associated transmembrane protein that is tyrosinephosphorylated upon fertilization. J Biol Chem 280(15):15029-15037

Sato K (2008) Signal transduction of fertilization in frog eggs and anti-apoptotic mechanism in human cancer cells: common and specific functions of membrane microdomains. Open Biochem J 2:49-59

Sato K, Aoto M, Mori K, Akasofu S, Tokmakov AA, Sahara S, Fukami Y (1996) Purification and characterization of a Src-related p57 protein-tyrosine kinase from Xenopus oocytes. Isolation of an inactive form of the enzyme and its activation and translocation upon fertilization. J Biol Chem 271(22):13250-13257

Sato K, Iwao Y, Fujimura T, Tamaki I, Ogawa K, Iwasaki T, Tokmakov AA, Hatano O, Fukami Y (1999) Evidence for the involvement of a Src-related tyrosine kinase in Xenopus egg activation. Dev Biol 209(2):308-320

Sato K, Tokmakov AA, Iwasaki T, Fukami Y (2000) Tyrosine kinase-dependent activation of phospholipase $\mathrm{C} \gamma$ is required for calcium transient in Xenopus egg fertilization. Dev Biol 224(2):453-469

Sato K, Ogawa K, Tokmakov AA, Iwasaki T, Fukami Y (2001) Hydrogen peroxide induces Src family tyrosine kinase-dependent activation of Xenopus eggs. Dev Growth Differ 43(1):55-72

Sato K, Iwasaki T, Sakakibara K, Itakura S, Fukami Y (2002a) Towards the molecular dissection of fertilization signaling: Our functional genomic/proteomic strategies. Proteomics 2(9):1079-1089

Sato K, Iwasaki T, Ogawa K, Konishi M, Tokmakov AA, Fukami Y (2002b) Low density detergentinsoluble membrane of Xenopus eggs: subcellular microdomain for tyrosine kinase signaling in fertilization. Development (Camb) 129(4):885-896

Sato K, Tokmakov AA, He CL, Kurokawa M, Iwasaki T, Shirouzu M, Fissore RA, Yokoyama S, Fukami Y (2003) Reconstitution of Src-dependent phospholipase C $\gamma$ phosphorylation and transient calcium release by using membrane rafts and cell-free extracts from Xenopus eggs. J Biol Chem 278(40):38413-38420

Sato K, Iwasaki T, Hirahara S, Nishihira Y, Fukami Y (2004) Molecular dissection of egg fertilization signaling with the aid of tyrosine kinase-specific inhibitor and activator strategies. Biochim Biophys Acta 1697(1-2):103-121

Sato K, Yoshino K, Tokmakov AA, Iwasaki T, Yonezawa K, Fukami Y (2006) Studying fertilization in cell-free extracts: focusing on membrane/lipid raft functions and proteomics. Methods Mol Biol 322:395-411

Shilling FM, Magie CR, Nuccitelli R (1998) Voltage-dependent activation of frog eggs by a sperm surface disintegrin peptide. Dev Biol 202(1):113-124

Simons K, Ikonen E (1997) Functional rafts in cell membranes. Nature (Lond) 387(6633): $569-572$

Simons K, Sampaio JL (2011) Membrane organization and lipid rafts. Cold Spring Harbor Perspect Biol 3(10):a004697

Simons K, Toomre D (2000) Lipid rafts and signal transduction. Nat Rev Mol Cell Biol 1(1): 31-39, Erratum in Nat Rev Mol Cell Biol 2(3):216

Swann K, Lai FA (2013) PLC $\zeta$ and the initiation of $\mathrm{Ca}^{2+}$ oscillations in fertilizing mammalian eggs. Cell Calcium 53(1):55-62

Thomas SM, Brugge JS (1997) Cellular functions regulated by Src family kinases. Annu Rev Cell Dev Biol 13:513-609

Wu W, Kinsey WH (2000) Fertilization triggers activation of Fyn kinase in the zebrafish egg. Int J Dev Biol 44(8):837-841 